\title{
Comparison of Adverse Effects of Newer Atypical Antipsychotics: An Evidence Based Review
}

\author{
Kharadi Dhruvika Gaurang ${ }^{1 *}$, Shah Agam Rajendra ${ }^{2}$, Ganguly Barna' \\ 'Department of Pharmacology, Pramukh Swami Medical College, Karamsad, Gujarat, INDIA. \\ ${ }^{2}$ Medical Affairs Division, Wockhardt Ltd., Mumbai, Maharashtra, INDIA
}

\begin{abstract}
Aim: To Compare the safety of asenapine and iloperidone as per information available in public domain. Method: In addition to their USFDA approved prescribing information, literature on these two drugs from Pubmed indexed journals in English language were searched for appropriate information. We found 8 relevant articles on asenapine and iloperidone, results of which are discussed in this review. Data on the adverse effects commonly reported for atypical antipsychotics were collected from these articles and compared. Descriptive statistics was used to present the results. Results: lloperidone is more frequently found to be associated with QTc prolongation (dose dependent), orthostatic hypotension \& related dizziness (despite slow up-titration), weight gain and antimuscarinic side effects. On other side, asenapine is more frequently associated with extrapyramidal side effects and akathisia as well as the only one associated with headache, oral hypoesthesia and adverse event related discontinuations. Conclusion: Although, asenapine appears to be less frequently associated with adverse events commonly associated with atypical antipsychotics,
\end{abstract}

complex sublingual dosing and higher discontinuation related to adverse events offsets this safety advantage. More importantly, both these agents require close counselling of patients for adequate compliance and/ or combination with other antipsychotic to achieve optimum antipsychotic efficacy in Indian context.

Key words: Adverse events, Antipsychotics, Asenapine, Iloperidone, Extrapyramidal side-effects, Indian context.

\section{Correspondence:}

Kharadi Dhruvika Gaurang, E/403, Gala Haven, Near Nirma University,

Off S.G. Highway, Ahmedabad - 382481, Gujarat, INDIA.

Phone no: 9925024779

Email: dgkharadi@yahoo.co.in

DOI: 10.5530/jyp.2017.9.29

\section{INTRODUCTION}

The "older" atypical antipsychotic drugs such as clozapine, olanzapine, quetiapine, risperidone, ziprasidone and aripiprazole were systematically reviewed for their safety profile in number of meta-analysis ${ }^{1,2}$ and research studies like CUTLASS $1^{3}$ \& CATIE. $^{4}$ The commonly reported adverse effects of these drugs include weight gain, extra pyramidal symptoms including tardive dyskinesia, antimuscarinic side effects, dyslipidemia, diabetes mellitus, prolactin elevation, QTc prolongation and sedation. ${ }^{5}$

Two atypical antipsychotics iloperidone and asenapine have been recently approved for acute treatment of schizophrenia by Drug Controller General (India) in February and April month of 2011, respectively. ${ }^{6}$ Both of them were claimed to be safer than older agents. However, there is no publication comparing two of them with each other in terms of their safety in Indian context. Therefore we conducted this study on safety comparison of these newer agents as per information freely available in public domain.

\section{MATERIALS AND METHODS}

This was an evidence based study on adverse effects of asenapine and iloperidone. Our interest in this subject started with an "Atypical Antipsychotic Drug Class Review Summary of Findings" published in The Oregon State Drug Review Newsletter of November 2011, ${ }^{7}$ which briefly reviewed the 'newest' atypical antipsychotics. The literature search strategy was to identify English language medical literature with appropriate information available as freely available full text journal articles indexed in PUBMED database. We found 8 relevant articles on asenapine ${ }^{8-10}$ and iloperidone ${ }^{11-15}$ results of which are discussed in this review. Prescribing information of asenapine ${ }^{16}$ ("SAPHRIS") and iloperidone ${ }^{17}$ ("FANAPT") were obtained from DailyMed website.

Data on the adverse effects commonly reported for atypical antipsychotics ${ }^{5}$ were collected from these articles and compared between the two drugs to identify their benefits and disadvantages over each other. It is pertinent to note here that we included safety data of only schizophrenia trials in this review as both products are approved only for that indication in India. ${ }^{6}$

Descriptive statistics was used to present the results.

\section{RESULTS}

\section{Weight gain}

Asenapine was associated with slightly higher weight gain than placebo with incidence of approximately $4.9 \%$ for gain of $\geq 7 \%$ weight from baseline with mean weight gain of around $1.1 \mathrm{~kg}$ over treatment periods up to 6 weeks. ${ }^{16}$ In long-term Clinical Trials, asenapine was associated with cumulatively higher incidence $14.7 \%$ for gain of $\geq 7 \%$ weight from baseline with mean weight gain of around $0.9 \mathrm{~kg}$ over treatment periods up to 52 weeks. ${ }^{16}$ In short term Clinical Trials, iloperidone was found to be associated with apparently higher weight gain than placebo with incidence of approximately $13 \%$ for gain of $\geq 7 \%$ weight from baseline with mean weight gain of around $2 \mathrm{~kg}$ over treatment periods up to 6 weeks, ${ }^{17}$ which appeared to be dose dependent. Long-term Clinical Trials demonstrated that most $(2 / 3)$ of weight gain with iloperidone appeared to happen during first 6 weeks indicating plateau effect in this effect with only small (1/3) amount of weight gain during rest of the 46 weeks of the 52 weeks

This is an open access article distributed under the terms of the Creative Commons Attribution-NonCommercial-ShareAlike 4.0 License, which allows others to remix, tweak, and build upon the work non-commercially, as long as the author is credited and the new creations are licensed under the identical terms. 
of treatment period leading to mean weight gain of $3.8 \mathrm{~kg} .{ }^{13}$ Results of weight gain obtained in short-term trials are shown in Table 1 and results obtained in long-term trials are shown in Table 3.

\section{Extra-pyramidal Symptoms}

In short term Clinical Trials up to 6 weeks, asenapine was found to be associated with approximately $6 \%$ incidence of akathisia and approximately $10 \%$ incidence of other EPS, both of which were apparently higher than that with placebo as well as appeared dose dependent. ${ }^{16}$ In long-term Clinical Trials of 30 weeks, asenapine was associated with apparently continuous reports of akathisia during both early $(3.6 \%$ in first 4 weeks) and late phase (6.2\% in last 26 weeks). ${ }^{9}$ In short term Clinical Trials up to 6 weeks, Iloperidone was associated with similar incidence of EPS as well as akathisia than placebo with incidence of approximately $3 \%$ \& $1 \%$, respectively. ${ }^{17}$ Long-term Clinical Trials of 52 weeks demonstrated that iloperidone was associated with small incidence (3.8\%) of akathisia and very small incidence $(<1 \%)$ of other EPS, showing apparent plateau of EPS after early phase of 6 weeks. ${ }^{14}$ Results of extra-pyramidal symptoms obtained in short term trials are described in Table 1 and results obtained in long-term trials are described in Table 3.

\section{Metabolic Side Effects}

In short term Clinical Trials up to 6 weeks, both asenapine as well as iloperidone were associated with small but apparently high increase in mean blood glucose levels $\left(3.2 \mathrm{mg} / \mathrm{dL}^{16}\right.$ as well as $\approx 7 \mathrm{mg} / \mathrm{dL},{ }^{17}$ respectively) compared to placebo. In long-term Clinical Trials up to 52 weeks, asenapine was once again associated with small increase in mean blood glucose levels $(2.4 \mathrm{mg} / \mathrm{dL}){ }^{16}$ whereas iloperidone was associated with reduction in blood glucose levels possibly due to reversal of levels increased with pre-trial antipsychotics. ${ }^{17}$ Results of blood glucose level changes were obtained in short term trials have been described in Table 2 and results obtained in long-term trials were described in Table 4.

In short term Clinical Trials up to 6 weeks, both asenapine as well as iloperidone were associated with small but apparently high increase in mean fasting serum levels of cholesterol $\left(0.4 \mathrm{mg} / \mathrm{dL}^{16}\right.$ as well as $\approx 8.1 \mathrm{mg} /$ $\mathrm{dL},{ }^{14}$ respectively) and low density lipoproteins - LDL $\left(1.3 \mathrm{mg} / \mathrm{dL}^{16}\right.$ as well as $9.03 \mathrm{mg} / \mathrm{dL},{ }^{17}$ respectively) compared to placebo. However, the changes in high density lipoproteins (HDL) and triglycerides (TG) were comparable to placebo. ${ }^{16,17}$ In long-term Clinical Trials up to 52 weeks, asenapine was associated with mild increase in mean fasting serum levels of cholesterol $(6 \mathrm{mg} / \mathrm{dL}){ }^{16}$ and LDL $(9.8 \mathrm{mg} / \mathrm{dL}),{ }^{16}$ whereas, iloperidone was associated with reduction in mean fasting serum levels of choles-

Table 1: Symptomatic Adverse events commonly associated with atypical antipsychotics in short term clinical trials of asenapine and iloperidone

\begin{tabular}{|c|c|c|c|c|c|c|c|c|}
\hline \multirow[b]{2}{*}{ Drugs } & \multicolumn{4}{|c|}{ Asenapine } & \multicolumn{4}{|c|}{ lloperidone } \\
\hline & Placebo & $5 \mathrm{mg}$ BID & $10 \mathrm{mg}$ BID & Overall & Placebo & $\begin{array}{l}\text { 10-16 mg/ } \\
\text { day }\end{array}$ & $20-24 \mathrm{mg} /$ day & Overall \\
\hline \multicolumn{9}{|l|}{ Weight Gain } \\
\hline$\geq 7 \%$ gain $(\%)$ & 1.6 & 4.4 & 4.8 & 4.9 & 4 & 12.8 & 18.2 & 13 \\
\hline Mean gain $(\mathrm{kg})$ & 0.1 & 1 & 0.9 & 1.1 & -0.1 & 2 & 2.7 & 2 \\
\hline Incidence (\%) of $\mathrm{AE}$ & $<1$ & 2 & 2 & 3 & 1 & 1 & 9 & - \\
\hline \multicolumn{9}{|l|}{ Extra-pyramidal Symptoms (EPS) } \\
\hline EPS (\%) & 7 & 9 & 12 & 10 & $4 \& 2^{*}$ & 5 & 4 & $3^{*}$ \\
\hline Akathisia (\%) & 3 & 4 & 11 & 6 & $2.7 \& 0^{*}$ & 1.7 & 2.3 & $1^{*}$ \\
\hline Tremor (\%) & - & - & - & - & 1.9 & 2.5 & 3.1 & - \\
\hline Additional Comments & \multicolumn{4}{|c|}{ Change in 3 EPS scales equivalent to placebo } & \multicolumn{4}{|c|}{ Anticholinergic use $9 \%$ equivalent to placebo $7.3 \%$} \\
\hline \multicolumn{9}{|l|}{ Antimuscarinic Side Effects } \\
\hline Dry mouth (\%) & 1 & 3 & 1 & 2 & 1 & 8 & 10 & - \\
\hline Constipation (\%) & 6 & 7 & 4 & 5 & - & - & - & - \\
\hline Tachycardia (\%) & - & - & - & - & 1 & 3 & 12 & - \\
\hline Blurred vision (\%) & - & - & - & - & 2 & 3 & 1 & - \\
\hline Dry mouth (\%) & 1 & 3 & 1 & 2 & 1 & 8 & 10 & - \\
\hline \multicolumn{9}{|c|}{ Orthostatic hypotension \& related side effects } \\
\hline Orthostatic hypotension (\%) & - & - & - & $<2$ & $1 \& 8.3^{@}$ & $3 \& 21.2^{@}$ & $5 \& 13.6^{@}$ & - \\
\hline Syncope (\%) & - & - & - & - & 0.2 & - & 0.4 & - \\
\hline Hypotension (\%) & - & - & - & - & $<1$ & $<1 \& 3.8^{@}$ & $3 \& 4.8^{@}$ & - \\
\hline Dizziness (\%) & 4 & 7 & 3 & 5 & $7^{\oplus} \& 2^{*}$ & $10^{\circledR}$ & $20^{\circledR} \& 7^{\star}$ & - \\
\hline \multicolumn{9}{|l|}{ Sleep related side effects } \\
\hline Somnolence (\%) & 7 & 15 & 13 & 13 & $5 \& 2.7^{@}$ & $9 \& 5.7^{@}$ & $15 \& 8^{@}$ & 11.9 \\
\hline Insomnia (\%) & 13 & 16 & 15 & 15 & - & - & - & - \\
\hline $\begin{array}{c}\text { Prolactin elevation related } \\
\text { events (\%) }\end{array}$ & 0 & - & - & 0.4 & $<1$ & 2 & 2 & - \\
\hline
\end{tabular}

\# 4-week fixed dose study; ${ }^{*}$ Short term trial compared to ziprasidone; \& @ haloperidol \& risperidone comparative study 
Table 2: Investigations related adverse events commonly associated with atypical antipsychotics in short term clinical trials of asenapine and iloperidone

\begin{tabular}{|c|c|c|c|c|c|c|c|c|}
\hline \multirow{2}{*}{ Drugs } & \multicolumn{4}{|c|}{ Asenapine } & \multicolumn{4}{|c|}{ lloperidone } \\
\hline & Placebo & $5 \mathrm{mg}$ BID & $10 \mathrm{mg}$ BID & Overall & Placebo & $10-16 \mathrm{mg} /$ day & $20-24$ mg/day & Overall \\
\hline \multicolumn{9}{|l|}{ Blood level changes } \\
\hline Glucose (mg/ dL) & -0.2 & 3.8 & 1.1 & 3.2 & $-0.5^{\#},-3.6 \& 3.2^{\star}$ & 9 & $6.6^{\#}, 16.2 \& 7.9^{*}$ & - \\
\hline Cholesterol (mg/ dL) & -2.2 & -2.4 & 3.3 & 0.4 & $-2.17^{\sharp},-7.7 \&-0.5^{\star}$ & - & $8.18^{\star}, 0.0 \& 8.1^{*}$ & - \\
\hline $\mathrm{LDL}(\mathrm{mg} / \mathrm{dL})$ & 0.1 & -0.2 & 2.6 & 1.3 & $-1.41^{\#}$ & - & $9.03^{*}$ & - \\
\hline $\mathrm{HDL}(\mathrm{mg} / \mathrm{dL})$ & 0.5 & 0.4 & 1 & 0.5 & $-3.35^{\#}$ & - & $0.55^{*}$ & - \\
\hline Triglycerides (mg/ dL) & -7.6 & -1.9 & 0.1 & 3.8 & $16.47^{\#},-35.4 \& 19.5^{*}$ & - & $-0.83^{\#},-26.5 \& 0.8^{*}$ & - \\
\hline Prolactin (ng/mL) & -10.7 & - & - & -6.5 & $-6.3^{*} \&-57.4$ & -23.1 & $2.6^{\#}$ & - \\
\hline Prolactin 4xULN (\%) & 0.6 & - & - & 2.6 & - & - & - & - \\
\hline \multicolumn{9}{|l|}{ Change in QTc Interval } \\
\hline QTc Change (msec) & 0 & - & - & $2-5$ & 0 & 3.9 & $9.1,11.4^{*} \& 19^{\$}$ & - \\
\hline
\end{tabular}

\# 4-week fixed dose study; ${ }^{*}$ Short term trial compared to ziprasidone; \& \$ along with metabolic inhibitors.

Table 3: Symptomatic adverse events commonly associated with atypical antipsychotics in long term clinical trials of asenapine and iloperidone

\begin{tabular}{|c|c|c|c|c|c|c|}
\hline \multirow[t]{2}{*}{ Drugs } & \multicolumn{3}{|c|}{ Asenapine } & \multicolumn{3}{|c|}{ lloperidone } \\
\hline & Early & Late & Overall & Early & Late & Overall \\
\hline \multicolumn{7}{|l|}{ Weight Gain } \\
\hline Mean gain $(\mathrm{kg})$ & - & - & $0.9(52 \mathrm{wk})$ & $2.6(6 \mathrm{wk})$ & $1.2(46 \mathrm{wk})$ & $3.8(52 \mathrm{wk})$ \\
\hline Other comments & \multicolumn{3}{|c|}{ Higher incidence with body mass index < 23 (22\%) vs. > 27 (9\%) } & \multicolumn{3}{|c|}{ More than $2 / 3$ of gain in first 6 weeks } \\
\hline Akathisia & $3.6(4 \mathrm{wk})^{*}$ & $6.2(26 \mathrm{wk})^{*}$ & - & $3.5(6 \mathrm{wk})$ & - & $3.8(52 \mathrm{wk})$ \\
\hline \multicolumn{7}{|l|}{ Antimuscarinic side effects } \\
\hline Dry mouth (\%) & $0.8^{*}(4 \mathrm{wk})$ & $2.3^{*}(26 \mathrm{wk})$ & - & - & - & - \\
\hline \multicolumn{7}{|c|}{ Orthostatic hypotension related side effects } \\
\hline Somnolence & $9.8^{*}(4 \mathrm{wk})$ & $13.7^{\star}(26 \mathrm{wk})$ & - & - & - & $2(52 \mathrm{wk})$ \\
\hline Insomnia & $11.1^{\star}(4 \mathrm{wk})$ & $18.9^{*}(26 \mathrm{wk})$ & - & - & - & $18.1(52 \mathrm{wk})$ \\
\hline Sedation & $3.6^{*}(4 \mathrm{wk})$ & $5.2^{*}(26 \mathrm{wk})$ & - & - & - & - \\
\hline $\begin{array}{l}\text { Prolactin elevation related } \\
\text { events }\end{array}$ & - & - & - & - & - & 0.3 (52 wk) \\
\hline
\end{tabular}

* Data from switchover trial versus olanzapine.

terol and LDL possibly due to reversal of levels increased with pre-trial antipsychotics. ${ }^{17}$ Results of lipid level changes obtained in short term trials are described in Table 2 and results obtained in long-term trials are described in Table 4.

\section{Prolactin Elevation}

In short term Clinical Trials up to 6 weeks, although asenapine was associated with reduction in mean prolactin levels $(6.5 \mathrm{ng} / \mathrm{mL})$ similar to placebo ${ }^{16}$ possibly due to reversal of levels increased with pre-trial antipsychotics, increase to level $\geq 4$ times upper limit of normal range in prolactin $(2.6 \%)$ as well as prolactin elevation related side effects $(0.4 \%)$ was seen in apparently higher proportion of subjects than placebo. ${ }^{16}$ On other hand, in short-term Clinical Trials, iloperidone was associated with decrease in prolactin levels, which was reduced with increase in dose of iloperidone compared to placebo suggesting possibly a dose dependent potential to elevate prolactin levels ${ }^{14,17}$ with slightly high incidence (2\%) of prolactin elevation related side effects in high dose groups. ${ }^{17}$ In longterm Clinical Trials of 52 weeks, asenapine was again associated with reduction in prolactin levels, ${ }^{16}$ whereas, for iloperidone data on prolactin levels was not available, but prolactin elevation related side effects were reported in small proportion ( 0.2 to $0.3 \%$ ) of patients. ${ }^{17}$ Results of prolactin level changes obtained in short term trials are described in Table 2 
Table 4: Investigation related adverse events commonly associated with atypical antipsychotics in long term clinical trials of asenapine and iloperidone

\begin{tabular}{|c|c|c|c|c|c|c|}
\hline \multirow[t]{2}{*}{ Drugs } & \multicolumn{3}{|c|}{ Asenapine } & \multicolumn{3}{|c|}{ lloperidone } \\
\hline & Early & Late & Overall & Early & Late & Overall \\
\hline \multicolumn{7}{|l|}{ Blood level changes } \\
\hline Glucose (mg/dL) & - & - & $2.4(52 \mathrm{wk})$ & -3.6 (3-6 mnth) & -9 (6-12 mnth) & -18 (>12 mnth) \\
\hline Cholesterol (mg/ dL) & - & - & $6(52 \mathrm{wk})$ & -19.4 (3-6 mnth) & -23.2 (6-12 mnth) & -19.4 (>12 mnth) \\
\hline $\mathrm{TG}(\mathrm{mg} / \mathrm{dL})$ & - & - & 9.8 (52 wk) & $-26.6(3-6 \mathrm{mnth})$ & -35.4 (6-12 mnth) & -17.7 (>12 mnth) \\
\hline Prolactin $(\mathrm{ng} / \mathrm{mL})$ & & & $-26.9(52 \mathrm{wk})$ & - & - & - \\
\hline \multicolumn{7}{|l|}{ Change in QTc Interval } \\
\hline QTc change (msec) & - & - & - & $3.2(6 \mathrm{wk})$ & - & $10.3(52 \mathrm{wk})$ \\
\hline
\end{tabular}

and results obtained in long-term trials are described in Table 4. Results of prolactin elevation related adverse events obtained in short term trials are described in Table 1 and results obtained in long-term trials are described in Table 3.

\section{QTc Prolongation}

In short term Clinical Trials, asenapine was associated with small (2 to 5 $\mathrm{msec}$ ) increase in mean QTc interval, ${ }^{16}$ whereas iloperidone was associated with significant increase in mean QTc interval (up to $11.4 \mathrm{msec}$ ), ${ }^{14}$ which appeared to be dose dependent as well as it was further increased by combining with metabolic inhibitors $(19 \mathrm{msec}) .{ }^{17}$ In long-term Clinical Trials of 52 weeks, iloperidone was again associated with increase in mean QTc interval, which was continuous during early $(3.2 \mathrm{msec}$ in first 6 weeks) and late phase ( $10.3 \mathrm{msec}$ at the end of 52 weeks). ${ }^{14}$ Results of QTc interval changes obtained in short term trials are described in Table 2 and results obtained in long-term trials are described in Table 4.

\section{Antimuscarinic Side Effects}

In short term Clinical Trials up to 6 weeks, asenapine was associated with antimuscarinic events of dry mouth (2\%) and constipation (5\%) at comparable level to placebo, ${ }^{16}$ whereas, iloperidone was associated with higher incidence of dry mouth $(\approx 9 \%)$ as well as tachycardia $(\approx 7.5 \%)$ compared to placebo that increased with dose, but the rate for blurring of vision (1\%) was comparable to placebo. ${ }^{17}$ On the contrary, in long-term Clinical Trials of 30 weeks, asenapine was associated with small proportion of patients reporting dry mouth (up to $2.3 \%$ ) which was continuously reported in both early ( $0.8 \%$ in first 4 weeks) and late $(2.3 \%$ in last 26 weeks) phase, possibly related to sublingual dosing rather than antimuscarinic side effects. ${ }^{9}$ Results of antimuscarinic side effects obtained in short term trials are described in Table 1 and results obtained in longterm trials are described in Table 3.

\section{Orthostatic Hypotension}

In short-term Clinical Trials up to 6 weeks, asenapine was associated slightly higher incidence of dizziness (5\%) than placebo, however the incidence of orthostatic hypotension was reported at low rate $(<2 \%)$ than placebo. ${ }^{16}$ In long-term Clinical Trials up to 30 weeks, asenapine was associated with fair incidence of dizziness (5.4\%), most of which was reported during first 4 weeks (4.4\%). ${ }^{9}$ On the contrary, in short-term Clinical Trials, compared to placebo, iloperidone was associated with higher incidence of orthostatic hypotension (up to $21.2 \%$ ), ${ }^{13}$ hypotension (up to $4.8 \%)^{13}$ and syncope $(0.4 \%),{ }^{17}$ as well as apparent higher incidence of dizziness (up to $20 \%$ ), ${ }^{13}$ which was dose dependent. Unfortunately data on orthostatic hypotension or related side effects was not available from long-term Clinical Trials of iloperidone. ${ }^{14}$ Results of orthostatic hypoten- sion and related side-effects obtained in short term trials are described in Table 1 and results obtained in long-term trials are described in Table 3.

\section{Sleep Related Side Effects}

In short-term Clinical Trials up to 6 weeks, both asenapine $(13 \%)^{16}$ and iloperidone $(11.9 \%)^{17}$ were associated with apparently high incidence of somnolence compared to placebo, which increased with dose for iloperidone, but not for asenapine. In short-term Clinical Trials, asenapine was also associated with high rate of insomnia $(15 \%)^{16}$ that was fairly similar to placebo, which might be related to the schizophrenia itself or other concomitant medications. In long-term Clinical Trials up to 52 weeks, iloperidone was associated with small incidence of somnolence $(2 \%),{ }^{14}$ but asenapine was associated with high incidence $(13.7 \%)$, most of which was reported in first 4 weeks $(9.8 \%) .{ }^{9}$ Both asenapine $(18.9 \%)^{9}$ and iloperidone $(18.1 \%)^{14}$ were associated with high incidence of insomnia, which was continuously reported in both early and late phase for asenapine. Results of sleep related side-effects obtained in short term trials are described in Table 1 and results obtained in long-term trials are described in Table 3.

\section{Other side effects}

Asenapine is also associated with headache in long term studies at $8.5 \%$ in first 4 weeks (early) and $14.5 \%$ in next 26 weeks (late). ${ }^{9}$ It is also associated with oral hypoesthesia related to sublingual dosing with placebo at $1 \%$, with $5 \mathrm{mg}$ BID at 6\%, with $10 \mathrm{mg}$ BID at 7\% (overall 5\%). ${ }^{16}$ Compared to iloperidone, which reported adverse event related discontinuation at the same rate as placebo, ${ }^{14}$ asenapine reported higher rate of adverse event related discontinuation than placebo (mostly related to somnolence, abdominal pain and nausea). ${ }^{9}$ A case series of 57 schizophrenic Indian patients treated with iloperidone ${ }^{15}$ reported nasal congestion as most frequent adverse event with incidence as high as $40.35 \%$.

\section{DISCUSSION}

Asenapine is only available as a sublingual tablet due to its high first pass metabolism (bioavailability is $35 \%$ sublingually $\&<2 \%$ orally), which makes it difficult to administer drug for patients of schizophrenia and mania. ${ }^{10}$ It is recommended as $5 \mathrm{mg}$ twice daily for the treatment of schizophrenia. Escalation to $10 \mathrm{mg}$ twice daily dosing is recommended after 1 week, only when tolerated. To maximise sublingual absorption, patients are recommended to allow asenapine tablet to dissolve in saliva and not to eat or drink for $10 \mathrm{~min}$ after its administration. ${ }^{7}$ Compliance to such dosing requirement appears quiet difficult during acute treatment of schizophrenia, unless prescriber appropriately consults the patients. In addition, side effects of oral hypoasthesia and paresthesia further add to the difficulty in achieving compliance. No wonder the product is report- 
ed to have numerically higher discontinuation rate than that of olanzapine in comparative switchover trial (36.6\% vs. $24.6 \%)$ linked to lack of efficacy ( $3 \%$ vs. $2.6 \%)$ and adverse events ( $15.8 \%$ vs. $10.6 \%) .{ }^{9}$

Prescriber should consider the risk of QTc prolongation associated with iloperidone while deciding to prescribe it. It has been recommended to be titrated slowly, starting with $1 \mathrm{mg}$ twice daily with daily dosage adjustment of $\leq 2 \mathrm{mg}$ twice daily to achieve target dose of 6 to $12 \mathrm{mg}$ twice daily, to avoid orthostatic hypotension due to its strong $a_{1}$ blocking properties. ${ }^{7}$ It certainly discourages to use it in acute treatment of schizophrenia as single agent due to such slow titration leading to delay of 1 to 2 weeks in controlling symptoms of schizophrenia, especially when published Clinical Trial blames such delay in therapeutic response for higher discontinuation rates in first 2 weeks. ${ }^{13}$ In Indian healthcare field, where delay in controlling symptoms frequently results in change of the treating doctor, it becomes obvious choice that iloperidone is mainly co-prescribed with multiple agents for the acute treatment of schizophrenia that compensates for such a delay.

In the safety comparison between the two, iloperidone was more frequently found to be associated with QTc prolongation (dose dependent), orthostatic hypotension \& related dizziness (despite slow up-titration as recommended), weight gain (apparently dose dependent) and antimuscarinic side effects (apparently dose-dependent). On other side, asenapine was more frequently associated with extra-pyramidal side effects and akathisia (apparently dose dependent) as well as the only one associated with headache, oral hypoesthesia (related to sublingual dosing) and adverse event related discontinuations.

Importantly in Indian context, both of these agents have met with higher discontinuation rates in early treatment due to complex dosing (sublingual dosing for asenapine ${ }^{9}$ and slow up-titration for iloperidone ${ }^{13}$ ) as well as delay in achieving optimum efficacy. Hence, while deciding to use any of these agents, prescriber must closely consult patients and/ or relatives about adequate compliance to achieve optimum efficacy of these products seen in controlled Clinical Trials. Alternately, author feels that these agents may be combined with other atypical antipsychotic or conventional antipsychotic during initial period to quickly achieve the therapeutic efficacy, followed by tapering them off when optimum efficacy for the newer products is achieved.

\section{CONCLUSION}

Amongst the two agents, asenapine appeared to be less frequently associated with adverse events commonly associated with atypical antipsychotics. But complex sublingual dosing and higher discontinuation related to adverse events compared to iloperidone offsets this safety advantage. Overall both these agents require close counselling of patients for adequate compliance and/ or combination with other antipsychotic to achieve optimum antipsychotic efficacy in Indian context.

\section{LIMITATIONS}

As this review is based on published evidence from Global Clinical Trials of asenapine and iloperidone, incidence of the adverse effects may differ in Indian patients from that reported in this article due to differences of genetic factors and ethnic perceptions. Hence, readers are requested to use this review as indicator of the differences between the two newer drugs.

\section{CONFLICT OF INTEREST}

No conflict of interest are declared.

\section{ABBREVIATION USED}

USFDA: United States Food \& Drug Administration; CUTLASS: Cost Utility of The Latest Antipsychotics in Schizophrenia Study; CATIE: Clinical Antipsychotics Trial of Intervention Effectiveness; EPS: Extra Pyramidal Side-effects; mg: Milligram; dL: Deciliter; HDL: High Density Lipoprotein; LDL: Low Density Liporpotein; TG: Trigylceride; BID: Twice Daily.

\section{REFERENCES}

1. Leucht S, Corves C, Arbter D, Engel RR, Li C, Davis JM. Second-generation versus first-generation antipsychotic drugs for schizophrenia: a meta-analysis. Lancet. 2009;373(9657):31-41.

2. Leucht S, Komossa K, Rummel-Kluge C, Corves $C$, Hunger H, Schmid F, et al. A meta-analysis of head-to-head comparisons of second-generation antipsychotics in the treatment of schizophrenia. Am J Psychiatry. 2009;166(2):152-63.

3. Jones, PB, Barnes TRE, Davies L, Dunn G, Lloyd H, Hayhurst KP, et al. Randomized Controlled Trial of the Effect on Quality of Life of Second vs. FirstGeneration Antipsychotic Drugs in Schizophrenia. Cost Utility of the Latest Antipsychotic Drugs in Schizophrenia Study (CUtLASS1). Arch Gen Psychiatry. 2006;63(10):1079-87.

4. Stroup TS, McEvoy JP, Swartz MS, Byerly MJ, Qlick ID, Canive JM, et al. The National Institute of Mental Health Clinical Antipsychotic Trials of Intervention Effectiveness (CATIE) Project: Schizophrenia Trial Design and Protocol Development. Schizophrenia Bulletin. 2003;29(1):15-31.

5. Sharif ZA. Overview of Safety and Tolerability of Atypical Antipsychotics Used in Primary Care. Primary Care Companion J Clin Psychiatry. 2003;5(3):14-21.

6. Central Drug Standards \& Control Organization. LIST OF APPROVED DRUG FROM 01-01-2011 to 31-05-2011. Cited on website http://www.cdsco.nic.in.

7. Hamer A. Atypical Antipsychotic Drug Class Review Summary of Findings: The Oregon State Drug Review. An Evidence Based Drug Therapy Resource November. 2011;1(1)

8. Potkin SG. Asenapine: a clinical overview. J Clin Psychiatry. 2011;72(1):14-18.

9. Cazorla P, Mackle M, Zhao J, Ha X, Szegedi A. Safety and tolerability of switching to asenapine from other antipsychotic agents: pooled results from two randomized multicenter trials in stable patients with persistent negative symptoms in schizophrenia. Neuropsychiatric Disease and Treatment. 2012;8:247-57.

10. Balraman R, Gandhi $H$. Asenapine, a new sublingual atypical antipsychotic. J Pharmacol and Pharmacother. 2010;1(1):60-1.

11. Citrome L. Iloperidone: A Clinical Overview. J Clin Psychiatry. 2011;72(1):19-23.

12. Weiden PJ. Iloperidone for the Treatment of Schizophrenia: An Updated Clinical Review. Clinical Schizophrenia \& Related Psychoses. 2012;6(1):34-44.

13. Citrome L. Iloperidone for schizophrenia: a review of the efficacy and safety profile for this newly commercialized second-generation antipsychotic. Int J Clin Pract. 2009;63(8):1237-48.

14. Caccia S, Pasina L, Nobil A. New atypical antipsychotics for schizophrenia: iloperidone. Drug Des Dev Ther. 2010;4:33-48.

15. Das S, Saha I, Mondal S, Bandyopadhyay S. Letter to the Editor: Nasal congestion with iloperidone therapy in a case series. J Young Pharm. 2013;5(3):108-9.

16. SAPHRIS ${ }^{\circledR}$ (Asenapine Maleate Sublingual Tablet) $5 \mathrm{mg}$ and $10 \mathrm{mg}$ [Prescribing Information] Whitehouse Station (NJ); Merck Sharp \& Dohme Corp.: Revised $10 / 2012$.

17. FANAPT ${ }^{\circledR}$ (Iloperidone) Tablets $1 \mathrm{mg}, 2 \mathrm{mg}, 4 \mathrm{mg}, 6 \mathrm{mg}, 8 \mathrm{mg}, 10 \mathrm{mg} \& 12 \mathrm{mg}$ [Prescribing Information] East Hanover, NJ: Novartis Pharmaceuticals Corporation: Revised January 2013.

Article History: Submission Date: 06-08-16 ; Received Date: 01-10-16; Acceptance Date: 02-01-17.

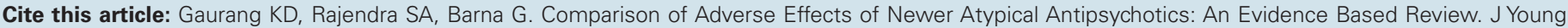
Pharm, 2017;9(2):140-4. 